\title{
Selective Activation and Functionalization of Linear Alkanes Initiated under Ambient Conditions by a Tungsten Allyl Nitrosyl Complex
}

\author{
Jenkins Y. K. Tsang, Miriam S. A. Buschhaus, Peter Legzdins*
}

Department of Chemistry, The University of British Columbia, Vancouver, British Columbia, Canada V6T $1 Z 1$

Supporting Material 


\section{Experimental Procedures}

General Methods. All reactions and subsequent manipulations involving organometallic reagents were performed under anaerobic and anhydrous conditions either under high vacuum or an inert atmosphere of prepurified dinitrogen. Purification of inert gases was achieved by passing them first through a column containing $\mathrm{MnO}$ and then a column of activated $4 \AA$ molecular sieves. Conventional glovebox and vacuum-line Schlenk techniques were utilized throughout. The gloveboxes utilized were Innovative Technologies LabMaster 100 and MS-130 BG dual-station models equipped with freezers maintained at $-30{ }^{\circ} \mathrm{C}$. Most of the reactions were performed in thick-walled glass vessels possessing Kontes greaseless stopcocks and side-arm inlets for vacuum-line attachment. Small-scale reactions and NMR spectroscopic analyses were conducted in J. Young NMR tubes which were also equipped with Kontes greaseless stopcocks. All solvents were dried with appropriate drying agents under a dinitrogen atmosphere and were distilled prior to use, or they were transferred directly under vacuum from the appropriate drying agent. Hydrocarbon solvents, diethyl ether, and HMDS were dried and distilled from sodium benzophenone ketyl. $\mathrm{CDCl}_{3}$ (CIL) was dried and distilled from calcium hydride. Pentane- $d_{12}$ (Aldrich) was stored over sodium metal in a resealable vessel and was subjected to 3 freezepump-thaw cycles before use. Commercially available $\left(\mathrm{CH}_{2}=\mathrm{CH}-\mathrm{CHMe}\right) \mathrm{MgCl}$ (Aldrich, $0.5 \mathrm{M}$ in THF) was transformed into the corresponding diallylmagnesium reagent in the usual manner. ${ }^{1}$ $\mathrm{Cp} * \mathrm{~W}(\mathrm{NO})\left(\mathrm{CH}_{2} \mathrm{CMe}_{3}\right) \mathrm{Cl}$ was prepared according to published procedures. ${ }^{2}$ The progress of most reactions was monitored by NMR spectroscopy, and the isolated yields of all new complexes have not been optimized. 
All IR samples were prepared as Nujol mulls, and their spectra were recorded on a Thermo Nicolet 4700 FT-IR spectrometer. NMR spectra were recorded at room temperature on a Bruker AV-400 spectrometer. All chemical shifts are reported in ppm, and all coupling constants are reported in $\mathrm{Hz} .{ }^{1} \mathrm{H}$ NMR spectra are referenced to the residual protio isotopomer present in a particular solvent, and ${ }^{13} \mathrm{C}$ NMR spectra are referenced to the natural-abundance carbon signal of the solvent employed. Where necessary, ${ }^{1} \mathrm{H}-{ }^{1} \mathrm{H}$ COSY, ${ }^{1} \mathrm{H}-{ }^{1} \mathrm{H}$ NOEDS, ${ }^{1} \mathrm{H}-{ }^{13} \mathrm{C}$ HMQC, ${ }^{1} \mathrm{H}^{-13} \mathrm{C}$ HMBC, and ${ }^{13} \mathrm{C}$ APT experiments were carried out to correlate and assign ${ }^{1} \mathrm{H}$ and ${ }^{13} \mathrm{C}$ NMR signals. Low-resolution mass spectra (EI, $70 \mathrm{eV}$ ) were recorded by the staff of the UBC mass spectrometry facility using a Kratos MS-50 spectrometer. Elemental analyses were performed by Mr. Minaz Lakha of the UBC microanalytical facility.

Preparation of $\mathrm{Cp}^{*} \mathbf{W}(\mathrm{NO})\left(\mathrm{CH}_{2} \mathrm{CMe}_{3}\right)\left(\eta^{3}-\mathrm{CH}_{2} \mathrm{CHCHMe}\right)(1)$. In a glovebox a 100$\mathrm{mL}$ Schlenk tube was charged with a magnetic stir bar and $\mathrm{Cp} * \mathrm{~W}(\mathrm{NO})\left(\mathrm{CH}_{2} \mathrm{CMe}_{3}\right) \mathrm{Cl}(1.00 \mathrm{~g}$, $2.20 \mathrm{mmol})$. A 350-mL Schlenk tube was then charged with a magnetic stir bar and $\left(\mathrm{CH}_{2} \mathrm{CHCHMe}\right)_{2} \mathrm{Mg} \cdot \mathrm{x}($ dioxane $)($ titre $=113.0 \mathrm{~g} / \mathrm{mol} \mathrm{R}, 0.25 \mathrm{~g}, 0.5$ equiv $)$. On a vacuum line, $\mathrm{Et}_{2} \mathrm{O}$ (approx. $40 \mathrm{~mL}$ and $60 \mathrm{~mL}$ ) was vacuum-transferred onto the $\mathrm{Cp} * \mathrm{~W}(\mathrm{NO})\left(\mathrm{CH}_{2} \mathrm{CMe}_{3}\right) \mathrm{Cl}$ and the diallylmagnesium reagents, respectively. The $\mathrm{Et}_{2} \mathrm{O}$ above the $\mathrm{Cp} * \mathrm{~W}(\mathrm{NO})\left(\mathrm{CH}_{2} \mathrm{CMe}_{3}\right) \mathrm{Cl}$ was allowed to melt, and this resulted in the formation of a purple solution which was kept cold with a dry ice/acetone bath. This purple solution was then cannulated dropwise into the $350-\mathrm{mL}$ Schlenk tube containing the magnesium reagent that was kept in a liquid $\mathrm{N}_{2}$ bath. The rate of addition was slow enough to allow the added solution to freeze upon contact with the frozen $\mathrm{Et}_{2} \mathrm{O}$. Additional $\mathrm{Et}_{2} \mathrm{O}(2 \times 10 \mathrm{~mL})$ was used to wash the $100 \mathrm{~mL}$-Schlenk tube to ensure quantitative transfer of the $\mathrm{Cp} * \mathrm{~W}(\mathrm{NO})\left(\mathrm{CH}_{2} \mathrm{CMe}_{3}\right) \mathrm{Cl}$ reactant. After the addition of the $\mathrm{Cp} * \mathrm{~W}(\mathrm{NO})\left(\mathrm{CH}_{2} \mathrm{CMe}_{3}\right) \mathrm{Cl}$ solution had been completed, the mixture was stirred for a further 45 
min while being maintained in the dry ice/acetone bath. The solution gradually turned brown, with the concomitant formation of a light brown suspension of $\mathrm{Mg}$ salts. The dry ice/acetone bath was then removed, and the solvent was evaporated from the final mixture in vacuo. Since complex 1 is thermally unstable, cold solvents $\left(-30{ }^{\circ} \mathrm{C}\right)$ had to be employed throughout its extraction and subsequent chromatography in order to minimize its decomposition. Thus, the crude product was extracted with pentane $(4 \times 50 \mathrm{~mL})$, and the extracts were transfered to the top of an alumina I column $(2 \times 5 \mathrm{~cm})$. The column was eluted with 3:1 pentane $/ \mathrm{Et}_{2} \mathrm{O}$. The orange-yellow eluate was collected, the solvents were removed in vacuo, and the residue was crystallized from 5:1 pentane/ $\mathrm{Et}_{2} \mathrm{O}$ at $-30{ }^{\circ} \mathrm{C}$ overnight to obtain $\mathbf{1}$ as orange-yellow crystalline clusters. The isolated solids were washed with small amounts of cold pentane $\left(-30{ }^{\circ} \mathrm{C}, 2 \times 5 \mathrm{~mL}\right)$ and then dried in vacuo. Yield $0.45 \mathrm{~g}(43 \%)$.

Characterization data for 1: IR $\left(\mathrm{cm}^{-1}\right) 1594\left(\mathrm{~s}, v_{\mathrm{NO}}\right)$. MS (LREI, $m / z$, probe temperature $\left.120{ }^{\circ} \mathrm{C}\right) 475\left[\mathrm{P}^{+},{ }^{184} \mathrm{~W}\right] .{ }^{1} \mathrm{H}$ NMR $\left(400 \mathrm{MHz}, \mathrm{C}_{6} \mathrm{D}_{6}\right) \delta 0.89\left(\mathrm{~d},{ }^{2} J_{\mathrm{HH}}=13.2,1 \mathrm{H}, \mathrm{CH}_{2} \mathrm{CMe}_{3}\right), 1.01$ (m, 1H, allyl CHMe), $1.32\left(\mathrm{~s}, 9 \mathrm{H}, \mathrm{CMe}_{3}\right), 1.48\left(\mathrm{~s}, 15 \mathrm{H}, \mathrm{C}_{5} \mathrm{Me}_{5}\right), 1.56\left(\mathrm{~d},{ }^{3} J_{\mathrm{HH}}=14.0,1 \mathrm{H}\right.$, allyl $\left.\mathrm{CH}_{2}\right), 1.59\left(\mathrm{~d},{ }^{2} J_{\mathrm{HH}}=13.2,1 \mathrm{H}, \mathrm{CH}_{2} \mathrm{CMe}_{3}\right), 1.89\left(\mathrm{~d},{ }^{3} J_{\mathrm{HH}}=6.0,3 \mathrm{H}\right.$, allyl Me $), 3.67\left(\mathrm{~d},{ }^{3} J_{\mathrm{HH}}=7.2\right.$ $1 \mathrm{H}$, allyl $\left.\mathrm{CH}_{2}\right), 4.97\left(\mathrm{ddd},{ }^{3} J_{\mathrm{HH}}=7.2,{ }^{3} J_{\mathrm{HH}}=9.4,{ }^{3} J_{\mathrm{HH}}=14.0,1 \mathrm{H}\right.$, allyl CH$) .{ }^{13} \mathrm{C}\left\{{ }^{1} \mathrm{H}\right\} \mathrm{NMR}(100$ $\left.\mathrm{MHz}, \mathrm{C}_{6} \mathrm{D}_{6}\right) \delta 9.5\left(\mathrm{C}_{5} \mathrm{Me}_{5}\right), 16.9$ (allyl Me), $27.9\left(\mathrm{CH}_{2} \mathrm{CMe}_{3}\right), 34.6\left(\mathrm{CH}_{2} \mathrm{CMe}_{3}\right), 39.3$ $\left(\mathrm{CH}_{2} \mathrm{CMe}_{3}\right), 52.4$ (allyl $\left.\mathrm{CHMe}\right), 74.3$ (allyl $\left.\mathrm{CH}_{2}\right), 106.0\left(C_{5} \mathrm{Me}_{5}\right), 114.6$ (allyl $\left.C \mathrm{H}\right)$. Anal. Calcd for $\mathrm{C}_{19} \mathrm{H}_{33} \mathrm{NOW}$ : C, 48.01; H, 7.00; N, 2.95. Found: C, 47.88; H, 7.32; N, 3.24.

Preparation of $\mathrm{Cp}^{*} \mathrm{~W}(\mathrm{NO})\left(n-\mathrm{C}_{5} \mathrm{H}_{11}\right)\left(\eta^{3}-\mathrm{CH}_{2} \mathrm{CHCHMe}\right)(2)$. In a glove box a sample of $1(60.0 \mathrm{mg}, 1.26 \mathrm{mmol})$ was dissolved in pentane in a 4-dram vial, and the solution was set aside. After $20 \mathrm{~h}$, the solution, which had darkened slightly in color, was transferred to the top 
of an alumina I column $(0.5 \times 5 \mathrm{~cm})$ supported by glass wool in a Pasteur pipette. The column was eluted with 3:1 pentane/ $\mathrm{Et}_{2} \mathrm{O}$, and the yellow band that developed was eluted and collected. The solvent was removed from the eluate in vacuo, and the oily residue was redissolved in pentane $(2 \mathrm{~mL})$. The solution was stored at $-30{ }^{\circ} \mathrm{C}$ overnight to induce to deposition of orange irregularly-shaped crystals of 2 (48 mg, 80\%).

Characterization data for 2: IR $\left(\mathrm{cm}^{-1}\right) 1597\left(\mathrm{~s}, \mathrm{v}_{\mathrm{NO}}\right)$. MS (LREI, $\mathrm{m} / z$, probe temperature $\left.120{ }^{\circ} \mathrm{C}\right) 475\left[\mathrm{P}^{+},{ }^{184} \mathrm{~W}\right] .{ }^{1} \mathrm{H}$ NMR (400 MHz, $\left.\mathrm{C}_{6} \mathrm{D}_{6}\right) \delta 1.04\left(\mathrm{t},{ }^{3} J_{\mathrm{HH}}=7.3,3 \mathrm{H}, n\right.$-pentyl Me), 1.07 (overlapping m, $1 \mathrm{H}, n$-pentyl $\mathrm{CH}_{2}$ ), 1.11 (overlapping m, $1 \mathrm{H}$, allyl $\left.\mathrm{CHMe}\right), 1.40(\mathrm{~m}, 2 \mathrm{H}, n$ pentyl $\mathrm{CH}_{2}$ ), 1.49 (obscured, $3 \mathrm{H}, n$-pentyl $\mathrm{CH}_{2}$ ), $1.50\left(\mathrm{~s}, 15 \mathrm{H}, \mathrm{C}_{5} \mathrm{Me}_{5}\right.$ ), 1.51 (obscured, $1 \mathrm{H}$, allyl $\left.\mathrm{CH}_{2}\right), 1.59\left(\mathrm{~m}, 1 \mathrm{H}, n\right.$-pentyl $\left.\mathrm{CH}_{2}\right), 1.93\left(\mathrm{~d},{ }^{3} J_{\mathrm{HH}}=5.8,3 \mathrm{H}\right.$, allyl Me), $2.08\left(\mathrm{~m}, 1 \mathrm{H}, n\right.$-pentyl $\left.\mathrm{CH}_{2}\right)$, $3.23\left(\mathrm{~d},{ }^{3} J_{\mathrm{HH}}=7.2,1 \mathrm{H}\right.$, allyl $\left.\mathrm{CH}_{2}\right), 4.89\left(\mathrm{ddd},{ }^{3} J_{\mathrm{HH}}=7.2,{ }^{3} J_{\mathrm{HH}}=9.6,{ }^{3} J_{\mathrm{HH}}=13.6,1 \mathrm{H}\right.$, allyl CH$)$. ${ }^{13} \mathrm{C}\left\{{ }^{1} \mathrm{H}\right\}$ NMR (100 MHz, $\left.\mathrm{C}_{6} \mathrm{D}_{6}\right) \delta 9.4\left(\mathrm{C}_{5} M e_{5}\right), 14.7$ (n-pentyl Me), 16.2 (n-pentyl $\left.\mathrm{CH}_{2}\right), 17.7$ (allyl Me), 23.0 (n-pentyl $\mathrm{CH}_{2}$ ), 34.0 (n-pentyl $\mathrm{CH}_{2}$ ), 39.3 (n-pentyl $\mathrm{CH}_{2}$ ), 54.1 (allyl $\mathrm{CHMe),}$ 74.4 (allyl $\left.\mathrm{CH}_{2}\right), 105.5\left(C_{5} \mathrm{Me}_{5}\right), 109.5$ (allyl $\mathrm{CH}$ ). Anal. Calcd for $\mathrm{C}_{19} \mathrm{H}_{33} \mathrm{NOW}$ : C, 48.01; $\mathrm{H}$, 7.00; N, 2.95. Found: C, 47.92; H, 6.91; N, 3.17.

The compound $\mathbf{C p} * \mathbf{W}(\mathbf{N O})\left(\boldsymbol{n}-\mathbf{C}_{5} \mathbf{D}_{11}\right)\left(\eta^{3}-\mathbf{C H}_{2} \mathbf{C H C H C H} \mathbf{H}_{2} \mathrm{D}\right)\left(\mathbf{2}-\boldsymbol{d}_{12}\right)$ was similarly prepared from 1 and $n-\mathrm{C}_{5} \mathrm{D}_{12}$. The ${ }^{1} \mathrm{H}$ NMR spectrum of $\mathbf{2}-\boldsymbol{d}_{12}$ displays similar features arising from the allyl ligand, with the exception that the methyl proton signal at $\delta 1.93$ now appears as a multiplet. The ${ }^{13} \mathrm{C}\left\{{ }^{1} \mathrm{H}\right\}$ NMR spectrum exhibits a 1:1:1 triplet at $17.5 \mathrm{ppm}\left({ }^{1} J_{\mathrm{CD}}=19 \mathrm{~Hz}\right)$, indicative of a deuterium atom havin been incorporated in the allyl methyl group. 
Preparation of $\mathbf{C p} * \mathbf{W}(\mathrm{NO})\left(\eta^{2}-\mathrm{CH}_{2}=\mathbf{C H}-\mathbf{C H}=\mathrm{CH}_{2}\right)\left(\mathrm{PMe}_{3}\right)(3)$. In a glove box a sample of $1(60.0 \mathrm{mg}, 1.26 \mathrm{mmol})$ was added to a small resealable vessel. At a vacuum line, an excess of $\mathrm{PMe}_{3}(2 \mathrm{~mL})$ was vacuum-transferred onto the solid, and the resulting yellow-orange solution was set aside. After $20 \mathrm{~h}$, the solution had lightened in color. The volatiles were then removed in vacuo, and the remaining solid was extracted with cold pentane $\left(-30^{\circ} \mathrm{C}, 5 \times 5 \mathrm{~mL}\right)$, leaving behind an unidentified white residue. The combined extracts were filtered through Celite supported on glass wool in a Pasteur pipette, and the filtrate was reduced in volume in vacuo. Storage of the sample at $-30{ }^{\circ} \mathrm{C}$ overnight led to the deposition of $\mathbf{3}$ as a yellow microcrystalline solid (25 mg, 41\%).

Characterization data for 3: IR $\left(\mathrm{cm}^{-1}\right) 1634\left(\mathrm{~s}, v_{\mathrm{NO}}\right)$. MS (LREI, $m / z$, probe temperature $\left.120{ }^{\circ} \mathrm{C}\right) 479\left[\mathrm{P}^{+},{ }^{184} \mathrm{~W}\right] .{ }^{1} \mathrm{H}$ NMR $\left(400 \mathrm{MHz}, \mathrm{C}_{6} \mathrm{D}_{6}\right) \delta 0.32\left(\mathrm{~m}, 1 \mathrm{H}\right.$, metal-bound $\left.H_{2} \mathrm{C}=\mathrm{CH}\right), 1.14$ $\left(\mathrm{d},{ }^{2} J_{\mathrm{HP}}=8.4,9 \mathrm{H}, \mathrm{PMe}_{3}\right), 1.59\left(\mathrm{~m}, 1 \mathrm{H}\right.$, metal-bound $\left.H_{2} \mathrm{C}=\mathrm{CH}\right), 1.65\left(\mathrm{~s}, 15 \mathrm{H}, \mathrm{C}_{5} \mathrm{Me}_{5}\right), 2.04(\mathrm{~m}$, $1 \mathrm{H}$, metal-bound $\left.\mathrm{H}_{2} \mathrm{C}=\mathrm{CH}\right), 4.70\left(\mathrm{dd},{ }^{2} J_{\mathrm{HH}}=2.0,{ }^{3} J_{\mathrm{HH}}=10.0,1 \mathrm{H}\right.$, uncoordinated $\left.H_{2} \mathrm{C}=\mathrm{CH}\right), 5.15$ $\left(\mathrm{dd},{ }^{2} J_{\mathrm{HH}}=2.0,{ }^{3} J_{\mathrm{HH}}=16.4,1 \mathrm{H}\right.$, uncoordinated $\left.H_{2} \mathrm{C}=\mathrm{CH}\right), 6.07\left(\mathrm{dt},{ }^{3} J_{\mathrm{HH}}=10.0,{ }^{3} J_{\mathrm{HH}}=16.4,1 \mathrm{H}\right.$, uncoordinated $\left.\mathrm{H}_{2} \mathrm{C}=\mathrm{C} H\right) .{ }^{13} \mathrm{C}\left\{{ }^{1} \mathrm{H}\right\} \mathrm{NMR}\left(100 \mathrm{MHz}, \mathrm{C}_{6} \mathrm{D}_{6}\right) \delta 10.1\left(\mathrm{C}_{5} M e_{5}\right), 16.3\left(\mathrm{~d},{ }^{1} J_{\mathrm{CP}}=32.0\right.$, $\mathrm{PMe}_{3}$ ), $31.4\left(\mathrm{~d},{ }^{2} \mathrm{~J}_{\mathrm{CP}}=12.0\right.$, metal-bound $\mathrm{H}_{2} \mathrm{C}=\mathrm{CH}$ ), 42.7 (metal-bound $\mathrm{H}_{2} \mathrm{C}=\mathrm{CH}$ ), 102.5 (uncoordinated $\mathrm{H}_{2} \mathrm{C}=\mathrm{CH}$ ), $103.1\left(C_{5} \mathrm{Me}_{5}\right), 149.7$ (uncoordinated $\mathrm{H}_{2} C=\mathrm{CH}$ ). Anal. Calcd. for $\mathrm{C}_{17} \mathrm{H}_{30} \mathrm{NOPW}$ : C, 42.61; H, 6.31; N, 2.92. Found: C, 42.67; H, 6.50; N, 2.72.

\section{Preparation of $\mathrm{Cp}^{*} \mathrm{~W}(\mathrm{NO})\left(n-\mathrm{C}_{7} \mathrm{H}_{15}\right)\left(\eta^{3}-\mathrm{CH}_{2} \mathrm{CHCHMe}\right)$ (4). Compound 4 was} prepared from compound $\mathbf{1}$ and $n$-heptane in a manner identical to that described above for the synthesis of compound $\mathbf{2}$ from $\mathbf{1}$ and $n$-pentane. Compound $\mathbf{4}$ was isolated as orange irregularlyshaped crystals in $42 \%$ yield. 
Characterization data for 4: IR $\left(\mathrm{cm}^{-1}\right) 1593\left(\mathrm{~s}, v_{\mathrm{NO}}\right)$. MS (LREI, $\mathrm{m} / z$, probe temperature $\left.120^{\circ} \mathrm{C}\right) 503\left[\mathrm{P}^{+},{ }^{184} \mathrm{~W}\right] .{ }^{1} \mathrm{H}$ NMR $\left(400 \mathrm{MHz}, \mathrm{C}_{6} \mathrm{D}_{6}\right) \delta 0.92\left(\mathrm{t},{ }^{3} \mathrm{~J}_{\mathrm{HH}}=7.2,3 \mathrm{H}, n\right.$-heptyl Me), 1.08 (overlapping m, $2 \mathrm{H}, n$-heptyl $\mathrm{CH}_{2}$ ), 1.10 (overlapping $\mathrm{m}, 1 \mathrm{H}$, allyl $\mathrm{CHMe}$ ), $1.30(\mathrm{~m}, 1 \mathrm{H}, n$ heptyl $\mathrm{CH}_{2}$ ), 1.36 (br m, 4H, $n$-heptyl $\mathrm{CH}_{2}$ ), 1.49 (obscured, $4 \mathrm{H}, n$-heptyl $\mathrm{CH}_{2}$ ), 1.51 (s, $15 \mathrm{H}$, $\mathrm{C}_{5} \mathrm{Me}_{5}$ ), 1.52 (obscured, $1 \mathrm{H}$, allyl $\left.\mathrm{CH}_{2}\right), 1.93\left(\mathrm{~d},{ }^{3} \mathrm{~J}_{\mathrm{HH}}=5.6,3 \mathrm{H}\right.$, allyl Me), $2.08(\mathrm{~m}, 1 \mathrm{H}, n$-pentyl $\left.\mathrm{CH}_{2}\right), 3.24\left(\mathrm{~d},{ }^{3} J_{\mathrm{HH}}=7.2,1 \mathrm{H}\right.$, allyl $\left.\mathrm{CH}_{2}\right), 4.90\left(\mathrm{ddd},{ }^{3} J_{\mathrm{HH}}=7.2,{ }^{3} J_{\mathrm{HH}}=9.2,{ }^{3} J_{\mathrm{HH}}=13.6,1 \mathrm{H}\right.$, allyl CH). ${ }^{13} \mathrm{C}\left\{{ }^{1} \mathrm{H}\right\}$ NMR (100 MHz, $\left.\mathrm{C}_{6} \mathrm{D}_{6}\right) \delta 9.4\left(\mathrm{C}_{5} M e_{5}\right), 14.4$ (n-heptyl Me), 16.4 (n-heptyl $\left.\mathrm{CH}_{2}\right)$, 17.7 (allyl Me), 23.3, 29.8, 32.7, 34.4, 38.0 (n-heptyl $\mathrm{CH}_{2}$ ), 54.1 (allyl $\mathrm{CHMe),} 74.4$ (allyl $\mathrm{CH}_{2}$ ), $105.5\left(C_{5} \mathrm{Me}_{5}\right), 109.5$ (allyl $\left.\mathrm{CH}\right)$.

Reaction Between Compound 2 and $\mathbf{I}_{2}$. In a glovebox, compound 2 (42.0 mg, 0.884 mmol) was dissolved in $\mathrm{CDCl}_{3}(3 \mathrm{~mL})$ in a resealable vessel containing a small stir bar. HMDS $(10 \mathrm{uL})$ was added via a microsyringe as an NMR integration standard. The ${ }^{1} \mathrm{H}$ NMR spectrum of a sample of the mixture was recorded, and the area under the doublet at $3.40 \mathrm{ppm}(1 \mathrm{H}$, allyl $\left.\mathrm{CH}_{2} \mathrm{CHCHCH}_{3}\right)$ was integrated against the singlet at $0.10 \mathrm{ppm}$ (18H, HMDS). The NMR sample was recombined with the rest of the solution, and the resealable vessel was attached to a vacuum line and maintained at $-60{ }^{\circ} \mathrm{C}$ with a dry ice/acetone bath. A solution of $\mathrm{I}_{2}(45.0 \mathrm{mg}, 2$ equiv) in $\mathrm{CDCl}_{3}(15 \mathrm{~mL})$ was prepared in a Schlenk tube, and the solution was added dropwise via a cannula to the resealable vessel maintained at $-60^{\circ} \mathrm{C}$. The Schlenk tube was finally rinsed with a small amount of cold $\mathrm{CDCl}_{3}(2 \times 2 \mathrm{~mL})$ to ensure quantitative transfer. The cold bath was removed after the addition of $\mathrm{I}_{2}$ was complete, and the reaction mixture was stirred for a further 1h. Overall the reaction mixture changed color from yellow-orange to dark greenish yellow. The resealable vessel was taken into a glove box, and a small amount of the reaction mixture was 
transferred into an NMR tube. The sample was analyzed by ${ }^{1} \mathrm{H}$ NMR spectroscopy, and integration of the area under the triplet at $3.20 \mathrm{ppm}\left(2 \mathrm{H}, \mathrm{CH}_{3} \mathrm{CH}_{2} \mathrm{CH}_{2} \mathrm{CH}_{2} \mathrm{CH}_{2} \mathrm{I}\right)$ against the HMDS signal revealed that $\mathrm{C}_{5} \mathrm{H}_{11} \mathrm{I}$ had been formed in approximately $73 \%$ yield.

The volatiles from the final reaction mixture were then vacuum-transferred into another Schlenk tube and were analyzed by ${ }^{1} \mathrm{H}$ NMR spectroscopy. Signals at $3.20 \mathrm{ppm}(\mathrm{t}, 2 \mathrm{H}$, $\left.\mathrm{CH}_{3} \mathrm{CH}_{2} \mathrm{CH}_{2} \mathrm{CH}_{2} \mathrm{CH}_{2} \mathrm{I}\right), 1.84$ ppm (m, 2H, $\left.\mathrm{CH}_{3} \mathrm{CH}_{2} \mathrm{CH}_{2} \mathrm{CH}_{2} \mathrm{CH}_{2} \mathrm{I}\right), 1.38 \mathrm{ppm}(\mathrm{m}, 4 \mathrm{H}$, $\mathrm{CH}_{3} \mathrm{CH}_{2} \mathrm{CH}_{2} \mathrm{CH}_{2} \mathrm{CH}_{2} \mathrm{I}$ and $\mathrm{CH}_{3} \mathrm{CH}_{2} \mathrm{CH}_{2} \mathrm{CH}_{2} \mathrm{CH}_{2} \mathrm{I}$ ) and $0.91 \mathrm{ppm}\left(\mathrm{t}, 3 \mathrm{H}, \mathrm{CH}_{3} \mathrm{CH}_{2} \mathrm{CH}_{2} \mathrm{CH}_{2} \mathrm{CH}_{2} \mathrm{I}\right)$ indicated the presence of 1-iodopentane, as confirmed by a ${ }^{1} \mathrm{H}$ NMR spectrum of an authentic sample. The volatiles were also analyzed by EI-MS. The presence of 1-iodopentane was indicated by a signal at $m / z=197.9\left[\mathrm{P}^{+}\right]$, while a signal at $m / z=181.9$ indicated the presence of at least one isomer of methallyl iodide.

The final organometallic residue was extracted with pentane $(2 \times 20 \mathrm{~mL})$. The extracts were filtered through Celite supported on a medium-porosity frit. The pentane was removed from the filtrate in vacuo, and the green-yellow residue was analyzed by ${ }^{1} \mathrm{H}$ NMR and IR spectroscopy. Spectroscopic data $\left[{ }^{1} \mathrm{H}\right.$ NMR in $\mathrm{CDCl}_{3}: \delta 2.18\left(\mathrm{C}_{5} \mathrm{Me}_{5}\right)$; IR (Nujol mull): $v 1630$ $\left.\mathrm{cm}^{-1}\left(v_{\mathrm{NO}}\right)\right]$ were consistent with the residue being principally the well known $\mathrm{Cp} * \mathrm{~W}(\mathrm{NO}) \mathrm{I}_{2}{ }^{3}$

X-ray Crystallography. Data collection for each compound was carried out at $-100 \pm 1$ ${ }^{\circ} \mathrm{C}$ on a Bruker X8 APEX diffractometer using graphite-monochromated Mo K $\alpha$ radiation.

Data for 1 were collected to a maximum $2 \theta$ value of $55.8^{\circ}$ in $0.5^{\circ}$ oscillations with $6.0 \mathrm{~s}$ exposures. The structure was solved by direct methods ${ }^{4}$ and expanded using Fourier techniques. All non-hydrogen atoms were refined anisotropically; hydrogen atoms H1a, H1b, H2 H3, H5a and $\mathrm{H} 5 \mathrm{~b}$ were refined isotropically, and all other hydrogen atoms were included in fixed 
positions. The final cycle of full-matrix least-squares analysis was based on 4599 observed reflections and 232 variable parameters.

Data for 2 were collected to a maximum $2 \theta$ value of $56.4^{\circ}$ in $0.5^{\circ}$ oscillations with $10.0 \mathrm{~s}$ exposures. The crystal was a two-component twin. The structure was solved by direct methods ${ }^{4}$ and expanded using Fourier techniques. All non-hydrogen atoms were refined anisotropically; hydrogen atoms $\mathrm{H} 1 \mathrm{a}, \mathrm{H} 1 \mathrm{~b}, \mathrm{H} 2$ and $\mathrm{H} 3$ were refined isotropically, and all other hydrogen atoms were included in fixed positions. The final cycle of full-matrix least-squares analysis was based on 4679 observed reflections and 222 variable parameters.

Data for 3 were collected to a maximum $2 \theta$ value of $55.8^{\circ}$ in $0.5^{\circ}$ oscillations with $10.0 \mathrm{~s}$ exposures. The structure was solved by direct methods ${ }^{4}$ and expanded using Fourier techniques. All non-hydrogen atoms were refined anisotropically; hydrogen atoms H1a, H1b, H2, H3, H4a and $\mathrm{H} 4 \mathrm{~b}$ were refined isotropically, and all other hydrogen atoms were included in fixed positions. The final cycle of full-matrix least-squares analysis was based on 4503 observed reflections and 222 variable parameters.

For each structure neutral-atom scattering factors were taken from Cromer and Waber. ${ }^{5}$ Anomalous dispersion effects were included in $F_{\text {calc }} ;{ }^{6}$ the values for $\Delta f^{\prime}$ and $\Delta f^{\prime}$ ' were those of Creagh and McAuley. ${ }^{7}$ The values for mass attenuation coefficients are those of Creagh and Hubbell. ${ }^{8}$ All calculations were performed using SHELXL-97. ${ }^{9}$ X-ray crystallographic data for all three structures are presented in Table 1. 
Table 1. X-Ray Crystallographic Data for Complexes 1, 2, and 3.

Complex

Empirical formula

Crystal Habit, color

Crystal size (mm)

Crystal system

Space group

Volume $\left(\AA^{3}\right)$

$\mathrm{a}(\AA)$

$\mathrm{b}(\AA)$

c $(\AA)$

$\alpha\left({ }^{\circ}\right)$

$\beta\left(^{\circ}\right)$

$\gamma\left({ }^{\circ}\right)$

Z

Density (calculated) $\left(\mathrm{Mg} / \mathrm{m}^{3}\right)$

Absorption coefficient $\left(\mathrm{cm}^{-1}\right)$

$\mathrm{F}_{000}$

Measured Reflections: Total

Measured Reflections: Unique

Final R Indices $^{\mathrm{a}}$

Goodness-of-fit on $\mathrm{F}^{2 \mathrm{~b}}$

Largest diff. peak and hole $\left(\mathrm{e}^{-} \AA^{-3}\right)$
1

\section{Crystal Data}

$\begin{array}{lll}\mathrm{C}_{19} \mathrm{H}_{33} \mathrm{NOW} & \mathrm{C}_{19} \mathrm{H}_{33} \mathrm{NOW} & \mathrm{C}_{17} \mathrm{H}_{30} \mathrm{NOPW} \\ \text { Prism, yellow } & \text { Rod, yellow } & \text { Prism, yellow } \\ 0.45 \times 0.30 \times 0.20 & 0.35 \times 0.20 \times 0.10 & 0.35 \times 0.20 \times 0.20 \\ \text { Monoclinic } & \text { Monoclinic } & \text { Monoclinic } \\ \mathrm{P} 2{ }_{1} / \mathrm{c} & \mathrm{P} 2{ }_{1} / \mathrm{n} & \mathrm{P} 2{ }_{1} / \mathrm{n} \\ 1940.63(8) & 1919.05(17) & 1875.15(16) \\ 9.0412(2) & 8.9209(5) & 8.1936(4) \\ 14.2430(3) & 18.9252(9) & 15.3514(8) \\ 15.6092(4) & 11.3669(6) & 14.9078(7) \\ 90 & 90 & 90 \\ 105.103(1) & 90.286(3) & 90.020(1) \\ 90 & 90 & 90 \\ 4 & 4 & 4 \\ 1.627 & 1.645 & 1.698 \\ 59.55 & 60.22 & 62.45 \\ 944 & 944 & 944\end{array}$

Data Collection and Refinement

$\begin{array}{lll}29663 & 36501 & 15837 \\ 4599 & 4679 & 4503 \\ \mathrm{R} 1=0.0178, & \mathrm{R} 1=0.0232, & \mathrm{R} 1=0.0216, \\ \mathrm{wR} 2=0.0423 & \mathrm{wR} 2=0.0526 & \mathrm{wR} 2=0.0536 \\ 1.080 & 1.038 & 1.014 \\ 1.185 \text { and }-0.806 & 1.619 \text { and }-0.774 & 1.579 \text { and }-0.932\end{array}$

${ }^{\mathrm{a}} \mathrm{R} 1$ on $\mathrm{F}=\Sigma\left|\left(\left|\mathrm{F}_{\mathrm{o}}\right|-\left|\mathrm{F}_{\mathrm{c}}\right|\right)\right| / \Sigma\left|\mathrm{F}_{\mathrm{o}}\right|\left(I_{\mathrm{o}}>2 \sigma I_{\mathrm{o}}\right) ; \mathrm{wR} 2=\left[\left(\Sigma\left(\mathrm{F}_{\mathrm{o}}{ }^{2}-\mathrm{F}_{\mathrm{c}}{ }^{2}\right)^{2}\right) / \Sigma \mathrm{w}\left(\mathrm{F}_{\mathrm{o}}{ }^{2}\right)^{2}\right]^{1 / 2}$ (all data); w $=[$ $\left.\sigma^{2} \mathrm{~F}_{\mathrm{o}}{ }^{2}\right]^{-1} ;{ }^{\mathrm{b}} \mathrm{GOF}=\left[\Sigma\left(\mathrm{w}\left(\left|\mathrm{F}_{\mathrm{o}}\right|-\left|\mathrm{F}_{\mathrm{c}}\right|\right)^{2}\right) / \text { degrees of freedom }\right]^{1 / 2}$. 


\section{References}

(1) Dryden, N. H.; Legzdins, P.; Trotter, J.; Yee, V. C. Organometallics 1991, 10, 2857.

(2) Debad, J. D.; Legzdins, P.; Batchelor, R. J.; Einstein, F. W. B. Organometallics 1993, 12, 2094.

(3) Dryden, N. H.; Legzdins, P.; Einstein, F. W. B.; Jones, R. H. Can. J. Chem. 1988, 66, 2100.

(4) SIR92: Altomare, A.; Cascarano, M.; Giacovazzo, C.; Guagliardi, A. J. Appl. Cryst. $1993,26,343$.

(5) Cromer, D. T.; Waber, J. T. International Tables for X-ray Crystallography; Kynoch Press: Birmingham, 1974; Vol. IV.

(6) Ibers, J. A.; Hamilton, W. C. Acta Crystallogr. 1964, 17, 781.

(7) Creagh, D. C.; McAuley, W. J. International Tables of X-ray Crystallography; Kluwer Academic Publishers: Boston, 1992; Vol. C.

(8) Creagh, D. C.; Hubbell, J. H. International Tables for X-ray Crystallography; Kluwer Academic Publishers: Boston, 1992; Vol. C.

(9) SHELXL97: Sheldrick, G. M. University of Göttingen, Germany, 1997. 